EPJ manuscript No.

(will be inserted by the editor)

\title{
The BMV experiment : a novel apparatus to study the propagation of light in a transverse magnetic field
}

\author{
Rémy Battesti ${ }^{1}$, Benoît Pinto Da Souza ${ }^{2}$, Sébastien Batut ${ }^{1}$, Cécile Robilliard ${ }^{2}$, Gilles Bailly ${ }^{2}$, Christophe Michel ${ }^{3}$, \\ Marc Nardone ${ }^{1}$, Laurent Pinard ${ }^{3}$, Oliver Portugall ${ }^{1}$, Gerard Trénec ${ }^{2}$, Jean-Marie Mackowski ${ }^{3}$, Geert L.J.A. Rikken ${ }^{1}$, \\ Jacques Vigué ${ }^{2}$ and Carlo Rizzo ${ }^{2}$ \\ 1 Laboratoire National des Champs Magnétiques Pulsés (UMR 5147, CNRS - INSA - Université Paul Sabatier Toulouse 3), \\ 31400 Toulouse cedex, France. \\ 2 Laboratoire Collisions Agrégats Réactivité (UMR 5589, CNRS - Université Paul Sabatier Toulouse 3), IRSAMC, 31062 \\ Toulouse Cedex 9, France. \\ 3 Laboratoire des Matériaux Avancés (LMA) (Université Claude Bernard Lyon 1, CNRS, IN2P3), Villeurbanne Cedex, France
}

Received: date / Revised version: date

\begin{abstract}
In this paper, we describe in detail the BMV (Biréfringence Magnétique du Vide) experiment, a novel apparatus to study the propagation of light in a transverse magnetic field. It is based on a very high finesse Fabry-Perot cavity and on pulsed magnets specially designed for this purpose. We justify our technical choices and we present the current status and perspectives.
\end{abstract}

PACS. PACS-key discribing text of that key - PACS-key discribing text of that key

\section{Introduction}

Experiments on the propagation of light in a transverse magnetic field date from the beginning of the 20th century. In 1901 Kerr 1 and in 1902 Majorana 2 discovered that linearly polarized light, propagating in a medium in the presence of a transverse magnetic field, acquires an ellipticity. In the following years, this magnetic birefringence has been studied in details by $\mathrm{A}$. Cotton and $\mathrm{H}$. Mouton 3 and it is known nowadays as the Cotton-Mouton effect. The velocity of light propagating in the presence of a transverse magnetic field $\mathrm{B}$ depends on the polarization of light, i.e. the index of refraction $n_{\|}$for light polarized parallel to the magnetic field is different from the index of refraction $n_{\perp}$ for light polarized perpendicular to the magnetic field. For symmetry reasons, the difference $\Delta n=\left(n_{\|}-n_{\perp}\right)$ is proportional to $B^{2}$. Thus, in general an incident linearly polarized light beam exits elliptically polarized from the magnetic field region. The ellipticity to be measured $\Psi$ can be written as

$$
\Psi=\pi \frac{L}{\lambda} \Delta n \sin 2 \theta
$$

where $L$ is the optical path in the magnetic field region, $\lambda$ the wavelength of the light, and $\theta$ the angle between light polarization and the magnetic field.

In dilute matter like gases, such an effect is usually very small and it needs very sensitive ellipsometers to be measured. Ab initio calculations can be performed using the

Send offprint requests to: carlo.rizzo@irsamc.ups-tlse.fr most advanced computational techniques and they still remain very challenging [4].

Propagation of light in vacuum in the presence of a transverse magnetic field has been experimentally studied since 1929 [5. The first motivation was to look for a magnetic moment of the photon. Only around 1970, thanks to the effective Lagrangian established in 1935 and 1936 by Kochel, Euler and Heisenberg 6] [7, it has been shown that the Cotton-Mouton effect should also exist in a vacuum [8] 9]. Quantum ElectroDynamics (QED) predicts that a field of $1 \mathrm{~T}$ should induce an anisotropy of the index of refraction of about $4 \times 10^{-24}$. This very fundamental prediction has not yet been experimentally verified.

Some of the earlier experiments were based on the use of an interferometer of the Michelson-Morley type. One of the two arms passed through a region where a transverse magnetic field was present inducing a difference in the light velocity that should have been observed as a phase shift [10] 11. In 1979, Iacopini and Zavattini 12 proposed to measure the ellipticity induced on a linearly polarized laser beam by the presence of a transverse magnetic field using an optical cavity in order to increase the optical path in the field. The effect to be measured was modulated in order to be able to use heterodyne technique to increase the signal to noise ratio.

In 1986, Maiani, Petronzio, and Zavattini [13] showed that hypothetical low mass, neutral, spinless boson, scalar or pseudoscalar, that couples with two photons could induce an ellipticity signal in the Zavattini apparatus similar to the one predicted by QED. Moreover, an appar- 
Please give a shorter version with: \authorrunning and \titlerunning prior to \maketitle

ent rotation of the polarization vector of the light could be observed because of conversion of photons into real bosons resulting in a vacuum magnetic dichroism which is absent in the framework of standard QED. The measurements of ellipticity and dichroism, including their signs, can in principle completely characterize the hypothetical boson, its mass $m_{a}$, the inverse coupling constant $M_{a}$, and the pseudoscalar or scalar nature of the particle. Maiani, Petronzio, Zavattini's paper was essentially motivated by the search for Peccei and Quinn's axions. These are pseudoscalar, neutral, spinless bosons introduced to solve what is called the strong CP problem [14. However, it was soon clear that such an optical apparatus could hardly exclude a range of axion parameters not already excluded by astrophysical bounds [15].

Following Zavattini's proposal, after tests at CERN in Geneva, Switzerland, [16], an apparatus has been set up at the Brookhaven National Laboratory, USA [17. No evidence for dichroism or for ellipticity induced by the magnetic field was found. The sensitivity being insufficient to detect the QED effect, only limits on the axion parameters could be published in 1993 [17, $M_{a}>2.8 \times 10^{6} \mathrm{GeV}$ at the $95 \%$ confidence level, provided $m_{a}<6.7 \times 10^{-3} \mathrm{eV}$.

In 1991, a new attempt to measure the vacuum magnetic birefringence has been started at the LNL in Legnaro, Italy, by the PVLAS collaboration [18. This experiment is again based on ref. 12. A Fabry-Perot cavity is used to increase the effect to be measured, while a superconductive $5 \mathrm{~T}$ magnet rotates around its own axis to modulate it. Eventually, the collaboration has published the observation of a magnetically induced dichroism in vacuum 19, and also of magnetically induced ellipticity in excess of what is expected according to QED [20]. These results have triggered a lot of interest in the field, in particular because the existence of axions could be the explanation for this unexpected signal 21. The proposed range is $2 \times 10^{5} \mathrm{GeV}<M_{a}<5.9 \times 10^{5} \mathrm{GeV}$, provided that $1 \times 10^{-3} \mathrm{eV}<m_{a}<1.5 \times 10^{-3} \mathrm{eV}$. Recently, the PVLAS collaboration has disclaimed their previous observations. An ellipticity signal is still present at $5.5 \mathrm{~T}$, while no ellipticity signal is observed at $2.3 \mathrm{~T}[22$. No clear explanation is given as to why the original signal has disappeared.

As soon as the original PVLAS results have become known, they have created big expectations, and the research field has gained a large momentum since this could be the first positive sighting of axions or axionlike particles 21. Indeed, the PVLAS result is in contradiction with the solar axion search, and in particular with the results of the CERN axion telescope CAST [23], which exclude axionlike particles with a mass smaller than $10^{-2} \mathrm{eV}$ and a inverse coupling constant smaller than $10^{10} \mathrm{GeV}$. To accommodate both results one should change the nature of axionlike particle interaction to justify their confinement to the interior of the sun (see e.g. 24]).

From the experimental point of view, different proposals have been put forward (see e.g. 25), most of them planning photoregeneration experiments. Such a kind of experiment is based on the idea that once an axionlike particle is created by photon conversion in a magnetic field, the particle escapes from the magnet region while the light beam can be easily confined. Now, if the created particle passes through another magnet region, it can be converted back into a photon that one can detect in a region where no photon should exist 26 . QED vacuum birefringence is also put forward in the proposal OSQAR 27. Most of these projects are expected to take data in 2007. First results of a photoregeneration experiment specially designed to test the PVLAS claim have appeared very recently [28] excluding the axionlike particle interpretation of the PVLAS original result with a confidence level greater than $99.9 \%$.

In the meantime new results have also been posted on the arXiv web site by the Q\&A (Quantum electrodynamics test \& search for Axion) project based at National Tsing Hua University, Taiwan. This project has started around 1996. The experimental set-up is similar to the PVLAS one. No effect has been detected, in contradiction with the original result from the PVLAS collaboration. As far as we understand, the confidence level is about $68 \%$ 29 .

In this paper, we describe a novel apparatus to study the propagation of light in a transverse magnetic field : the BMV (Biréfringence Magnétique du Vide) experiment. It is based on a very high finesse Fabry-Perot cavity and on a pulsed transverse magnet specially designed for this purpose. We justify our technical choices and we present the current status and perspectives. We show that our apparatus has the sensitivity to test the PVLAS result, and eventually to measure the vacuum magnetic birefringence. A very important $Q E D$ prediction that has been waiting to be tested since about 75 years.

\section{The optical apparatus}

In fig. 11 we present a sketch of our optical apparatus. The optical cavity and the laser source are put on two different optical tables, linked by a polarization maintaining optical fiber (PM fiber).

On the first table, light emitted by a mono-mode frequency controlled Nd:YAG laser at a wavelenght $\lambda=1064$ $\mathrm{nm}$ passes through a Faraday isolator FI.

A telescope $\mathrm{T} 1$ adapts the beam waist to be injected into the fiber. Mirrors M1 and M2 transport the beam to a $\lambda / 2$ waveplate and a polarizer prism P2 which allow to adjust beam intensity at the fiber entrance. Fiber collimator Fin launches light into the fiber with a coupling efficiency of about $85 \%$.

Light exits by the collimator Fout on the second table and it passes through an electro-optical modulator EO. This modulator is part of a feedback loop designed to lock the beam polarization. This loop is constituted by a beamsplitter BS that sends a fraction of light to a polarizer prism P3, a photodiode PdP detects the light transmitted by P3. The signal of PdP drives a locking circuit PolLC that maximizes the transmission through P3 by acting on the EO modulator.

Light going through the beamsplitter BS is matched to the cavity waist by a telescope $\mathrm{T} 2$ and transported by 
mirrors M3 and M4. These two mirrors are also used to align the optical beam with the optical axis of the FabryPerot cavity made by two very high reflectivity mirrors CM1 and CM2.

A system of high precision translations and rotations for the Fabry-Perot cavity mirrors and also for the polarizers has been designed and assembled. The piezoelectric stacks of the mirror alignment system as well as the mechanism allowing their rotation are adapted to ultra-high vacuum.

Before entering the Fabry-Perot cavity light is polarized by the polarizer P. Polarization of the light transmitted by the cavity is analyzed by the analyzer A. The extraordinary ray is detected by photodiode PdT, while the ordinary ray after being reflected by a mirror M5 is detected by photodiode PdI0. Both signals are used in the data analysis. Mirror M5 turns with prism A and extraction of the ordinary ray can be done at any position of A.

All the optical components from the polarizer $\mathrm{P}$ to the analyzer $\mathrm{A}$ are placed in a ultra high vacuum chamber not shown in the figure.

Light reflected back by the Fabry-Perot cavity arrives at the beamsplitter BS and it is sent to the photodiode $\mathrm{PdR}$, the signal of which is used to drive the phase locking circuit PhLC that locks the laser frequency to the FabryPerot cavity.
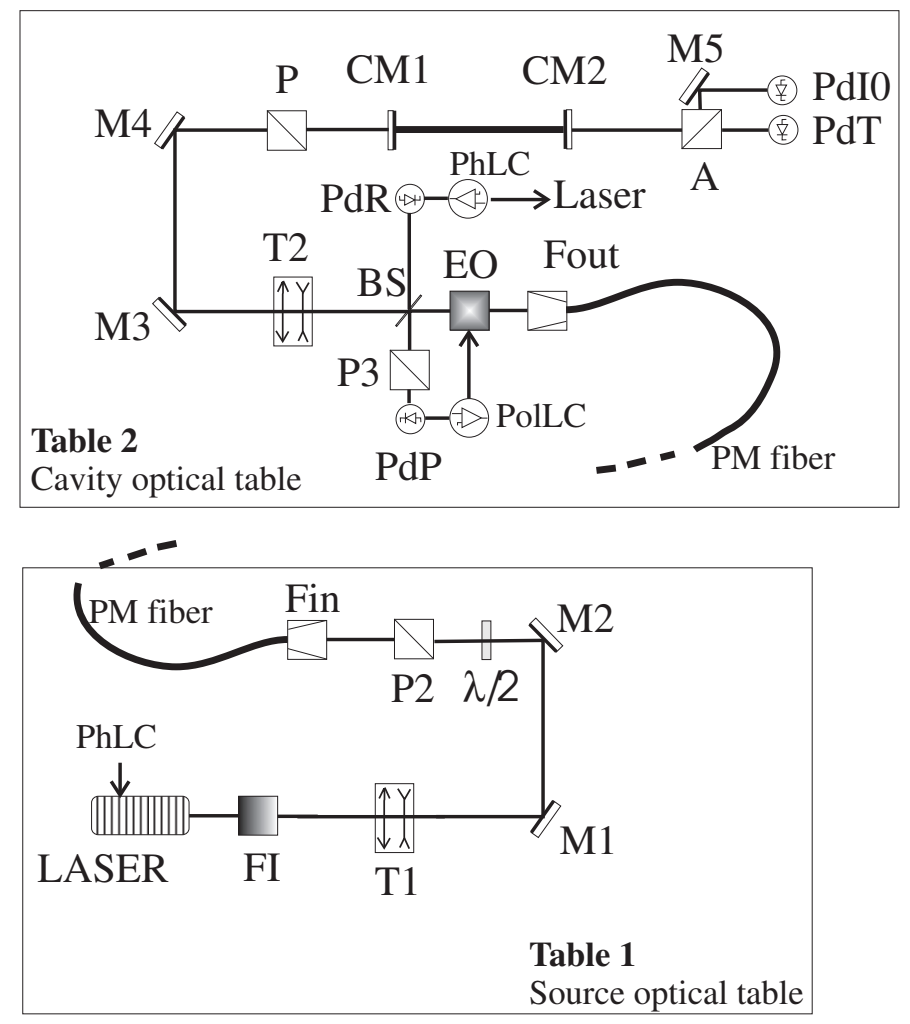

Fig. 1. Scheme of the optical apparatus.

\subsection{The Fabry-Perot Cavity}

Our Fabry-Perot cavity is made by two identical mirrors of $25.4 \mathrm{~mm}$ (1 inch) diameter. The length of the cavity $\mathrm{D}$ in the final version of our setup should not exceed 2.5 meters. The radius of curvature $\mathrm{C}$ of the mirrors has been chosen equal to $8 \mathrm{~m}$. The resonant frequencies of the cavity modesis given by the following formula

$$
\nu_{q l m}=\left[q+(l+m+1) \frac{\arccos \left(1-\frac{D}{C}\right)}{\pi}\right] \frac{c}{2 D}
$$

We want that only one mode resonates at the frequency of the $T E M_{00}$ mode, thus

$$
\frac{\arccos \left(1-\frac{D}{C}\right)}{\pi} \neq \frac{p}{k+1}
$$

where $p$ and $k$ are integers. Choosing $C=8 \mathrm{~m}$ guarantees that only modes with $l+m \gg 1$ can not fulfill eq. 3 .

The minimum waist $w_{0}$ is situated at the center of the cavity and it is given by eq. 4

$$
w_{0}=\sqrt{\left(\frac{\lambda}{2 \pi}\right) \sqrt{D(2 C-D)}}
$$

On the mirror the waist $w_{m}$ is given by the following formula :

$$
w_{m}=\sqrt{\left(\frac{\lambda}{2 \pi}\right) \sqrt{\frac{4 C^{2} D}{2 C-D}}}
$$

For $C=8 \mathrm{~m}$ and $D=2.5 \mathrm{~m}$, we get $w_{0} \simeq 1 \mathrm{~mm}$ and $w_{m} \simeq 1.02 \mathrm{~mm}$.

Assuming a good mode matching, cavity transmission depends on the mirror transmissivity $T$ and mirror losses $P$. The ratio $r_{I}$ between the intensity of the transmitted light $I_{t}$ and the intensity of the incident light can be written as

$$
r_{I}=\frac{I_{t}}{I_{i}}=\left(\frac{T}{T+P}\right)^{2}
$$

The value of the sum $T+P$ is fixed by the value of the requested finesse since $F=\frac{\pi}{1-R}$ where the mirror reflectivity $R$ is equal to $1-(T+P)$. For having a finesse between 500000 and 1000000 , let's assume that $P=$ $3 \times 10^{-6}$ and $T=2 \times 10^{-6}$. In this case $r_{I} \simeq 0.16$.

Let's assume in the following that we want a shot noise limited sensitivity $\Psi_{s}$ of the order of $10^{-9} \mathrm{rad} \mathrm{Hz}^{-1 / 2}$. As we show in the following (see eq. 31), assuming a quantum efficiency of the photodiode $q=0.7$, one finds that $I_{t}$ has to be about $40 \mathrm{~mW}$. If $r_{I} \simeq 0.12$, the incidence intensity $I_{i}$ has to be about $250 \mathrm{~mW}$.

Now, the intensity $I_{m}$ on the surface of the exit mirror of the cavity is $I_{t} / T$ i.e. about $20 \mathrm{~kW}$. This corresponds to a power density of the order of $600 \mathrm{~kW} / \mathrm{cm}^{2}$. 
Mirrors are always unintentionally slightly birefringent (see e.g. [34]) and therefore the Fabry-Perot cavity resonance line will be separated in two. Light polarized as the fast axis of birefringence will see a different optical path with respect to the light polarized as the slow axis of birefringence. In ref. 35] the issue of a birefringent FabryPerot cavity in the framework of ellipticity measurements has been discussed in detail. We just recall here that the frequency separation of the two polarizations is given by

$$
\Delta \nu_{\delta}=\frac{\delta}{2 \pi} \frac{c}{2 D}
$$

where $\delta$ is the mirror birefringence. The line width is

$$
\Delta \nu=\frac{1}{F} \frac{c}{2 D}
$$

To avoid that the cavity acts as polarizer prism, it is necessary that $\Delta \nu_{\delta}<\Delta \nu$, thus

$$
\delta<\frac{2 \pi}{F}
$$

Thus for a 1000000 finesse cavity $\delta<6.28 \times 10^{-6}$, which is demanding but which has already been observed for high finesse mirrors 34 .

\subsubsection{Magnetic field modulation and cavity finesse}

In our project, we plan to use a very high finesse cavity to increase the ellipticity signal to be measured. For 1000 000 cavity finesse and 2 meters cavity length, the photon lifetime in the cavity $\tau=F D /(\pi c)$ is about $2 \mathrm{msec}$. This duration is not negligible with respect to the magnetic pulse duration.

In the following, we calculate the output field of a Fabry-Perot interferometer in the case where the optical path is a slowly varying function of time. More precisely, the phase acquired by the light wave while going forward and backward in the Fabry-Perot cavity is given by:

$$
\psi=2 k D+\phi(t)
$$

We assume that $\phi(t)$ has negligible variations over the time $t_{D}=D / c$ taken by the light wave to go from one mirror to the other. However, because the cavity has a very high finesse $F$, the variations of $\phi(t)$ over the photon lifetime in the cavity $\tau$ are not necessarily negligible and the goal of the calculation is to express how the photon lifetime averages the phase $\phi(t)$. It seems that there is no general solution for the field exiting from a Fabry-Perot interferometer which is not stationary in time. The present calculation is approximate and assumes that the phase $\phi(t)$ is very small $(\phi(t)$ could be large but the varying part must be small and we assume that there is no time independent part).

We calculate the light field exiting from the FabryPerot at the instant $t$, by summing the contributions of the rays which have been transmitted with $n$ return paths:

$$
\begin{gathered}
\frac{E_{\text {out }}(t)}{T E_{\text {in }} \exp \left[i \phi\left(t-\left(t_{D}\right)\right)\right]}= \\
\sum_{j=0}^{\infty} R^{j} \exp \left[i\left(2 j k D+\sum_{p=1}^{j} \phi\left(t-2 p t_{D}\right)\right)\right]
\end{gathered}
$$

We assume that the amplitude reflection (respectively transmission) coefficients of the two mirrors are real and we note their product as $R$ (respectively $T$ ). The incident field $E_{i n}$ is taken as perfectly monochromatic. The light which has been reflected $n$ times at both ends of the FabryPerot interferometer has sampled the phase shift $\phi(t)$ at the times $\left(t-2 p t_{D}\right)$ with $p$ varying from 1 to $j$. For the general case, this expression cannot be evaluated analytically.

We assume that the Fabry-Perot cavity is at resonance $(2 k D=0[2 \pi])$ and that the phase $\phi(t)$ is very small so that we can replace the exponential by its first-order expansion:

$$
\exp \left[i \sum_{p=1}^{j} \phi\left(t-2 p t_{D}\right)\right] \approx 1+i \sum_{p=1}^{j} \phi\left(t-2 p t_{D}\right)
$$

The condition is not only $\phi(t) \ll 1$ but because the sum extends over a number of terms comparable to the finesse $F$, we must have $F \phi(t) \ll 1$. We must calculate two sums:

$$
\begin{gathered}
\sum_{j=0}^{\infty} R^{j}=\frac{1}{1-R} \\
\sum_{j=1}^{\infty} R^{j} \sum_{p=1}^{j} \phi\left(t-2 p t_{D}\right)= \\
\sum_{p=1}^{\infty} \phi\left(t-2 p t_{D}\right) \sum_{j=p}^{\infty} R^{j}= \\
\sum_{p=1}^{\infty} \phi\left(t-2 p t_{D}\right) \frac{R^{p}}{1-R}
\end{gathered}
$$

We can rewrite $R^{p}$ :

$$
R^{p}=\exp \left[-(\Gamma / 2) p\left(2 t_{D}\right)\right]
$$

where $\Gamma=1 / \tau \approx(1-R) / t_{D}$ and we get:

$$
\begin{gathered}
\sum_{j=1}^{\infty} R^{j} \sum_{p=1}^{j} \phi\left(t-2 p t_{D}\right)= \\
\sum_{p=1}^{\infty} \phi\left(t-2 p t_{D}\right) \exp \left[-(\Gamma / 2) p\left(2 t_{D}\right)\right] \frac{1}{1-R} \approx \\
\frac{1}{(1-R)^{2}} \int_{0}^{+\infty}(\Gamma / 2) \frac{d t^{\prime}}{2} \phi\left(t-t^{\prime}\right) \exp \left[-(\Gamma / 2) t^{\prime}\right]
\end{gathered}
$$

The final result is that the output field is given by: 


$$
\frac{E_{\text {out }}(t)}{T E_{\text {in }} \exp \left[i \phi\left(t-t_{D}\right)\right]} \approx \frac{1}{1-R}\left[1+i \frac{\langle\phi\rangle}{2(1-R)}\right]
$$

where

$$
\langle\phi\rangle=\int_{0}^{+\infty}(\Gamma / 2) d t^{\prime} \phi\left(t-t^{\prime}\right) \exp \left[-(\Gamma / 2) t^{\prime}\right]
$$

It is easy to make $\phi(t)=0$ in the result and then we find the traditional result of the Fabry Perot cavity at resonance:

$$
\frac{E_{\text {out }}(t)}{T E_{\text {in }}}=\frac{1}{1-R}
$$

For the sake of simplicity, let's assume that $B\left(t^{\prime}\right)=$ $B_{0} \sin \left(\omega t^{\prime}\right) . B\left(t^{\prime}\right)^{2}$ can be written as

$$
B\left(t^{\prime}\right)^{2}=\frac{B_{0}^{2}}{4}\left[2-e^{\left(2 i \omega t^{\prime}\right)}-e^{\left(-2 i \omega t^{\prime}\right)}\right]
$$

and

$$
\langle\phi\rangle \propto \int_{0}^{\infty} \frac{B_{0}^{2}}{4}\left[2-e^{(2 i \omega(t-\theta))}-e^{(-2 i \omega(t-\theta))}\right] e^{(-(\Gamma / 2) \theta)} d \theta
$$

where $\theta=t-t^{\prime}$. Finally, we obtain that

$$
\langle\phi\rangle \propto B_{0}^{2}\left[1-\frac{1}{\sqrt{1+\left(\frac{2 \omega}{\Gamma / 2}\right)^{2}}} \cos (2 \omega t+\varphi)\right]
$$

with $\tan \varphi=-\frac{2 \omega}{\Gamma}$

Thus when the magnetic field varies during the lifetime of the photons in the cavity, the ellipticity acquired by the light depends on an attenuated averaged value of the square of the magnetic field and moreover the ellipticity is not in phase with the square of the field. To avoid such an effect, it is clear that one needs

$$
\frac{2 \omega}{\Gamma / 2} \ll 1
$$

In our experiment, special care will be devoted to minimize such an effect.

\subsection{Cavity mirrors}

To increase as much as possible our signal to noise ratio, we need a cavity with a very high finesse. As far as we know, the highest finesse ever published is about $2 \times 10^{6}$ [36, while the highest quality factor $Q$ is the one of the PVLAS cavity [19], $Q \simeq 4 \times 10^{12}$ corresponding to a cavity linewidth of about $200 \mathrm{~Hz}$ and a storage time of $0.5 \mathrm{~ms}$.

Our cavity mirrors are made by the Laboratoire des Matériaux Avancés (LMA). Thanks to their know-how we have currently at our disposal 4 mirrors with losses ranging from 3 to $9 \mathrm{ppm}$ and transmission ranging from 1.5 to $1.9 \mathrm{ppm}$. This corresponds to finesses ranging from 300 000 to 700000 . The main problem is to handle these mirrors in an adequate way because they are very sensitive to pollution. Therefore we have built a special clean room with laminar flow cabinets for their manipulation. The access to this room is limited to fully equipped personnel (see fig 22).

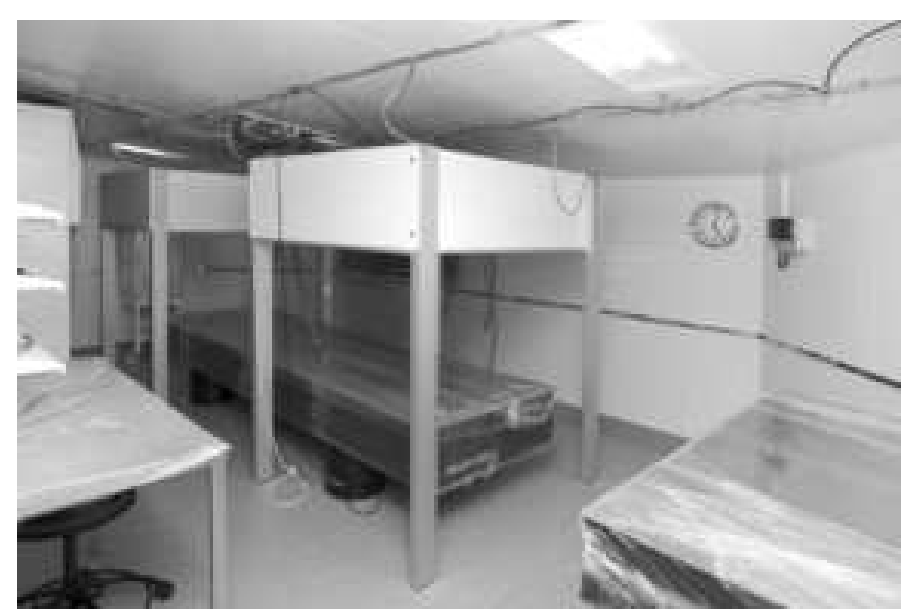

Fig. 2. Photo of the experimental room.

The mirrors are put in a home made kinematic mount which is activated by two piezoelectric wedges. They allow us to align the mirror reflecting surface perpendicular to the cavity axis with a precision better than $4 \mu \mathrm{rad}$. This aligning system operates in ultra high vacuum. Finally, we use a bronze wheel for rotating the mirror mount to minimize the cavity birefringence. It allows us a rotation smaller than 1 mrad.

\subsection{Laser locking to the Fabry-Perot cavity}

The laser source is locked to the Fabry-Perot cavity to maximize the injection of the light into the magnetic field region using the usual Pound-Drever-Hall technique [37. The laser crystal itself is used to phase modulate the laser light 38 .

The light source is a tuneable non planar ring oscillator Nd:YAG laser emitting $\sim 200 \mathrm{~mW}$ of power at a wavelength $\lambda=1064 \mathrm{~nm}\left(\nu=2.82 \times 10^{14} \mathrm{~Hz}\right)$. The frequency of the laser can be changed by two methods: a fast one (bandwidth $>10 \mathrm{kHz}$ ) based on a piezoelectric actuator acting on the laser crystal and a slow one (bandwidth $<1 \mathrm{~Hz}$ ) based on the change of the crystal temperature. In practice, the former allows to vary the laser frequency of about $50 \times 10^{6} \mathrm{~Hz}$, whereas the latter yields changes of about $100 \times 10^{9} \mathrm{~Hz}$.

In fig. 3 we show a simplified scheme of our locking circuit.

The novelty, compared for example to ref. [39], is that we control the laser frequency using three different feedback signals instead of two. As usual, a very low band- 


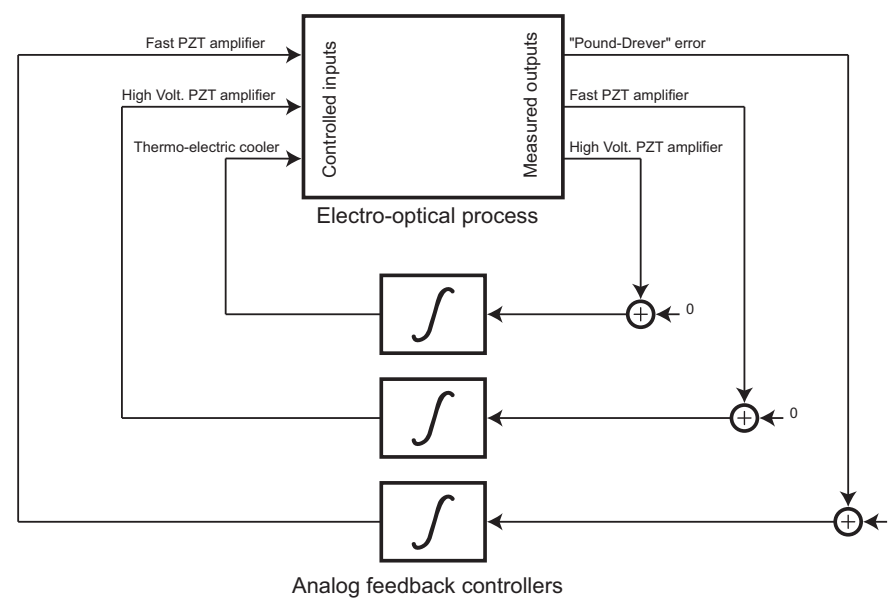

Fig. 3. Conceptual design of our locking circuit.

width one acts on the crystal temperature, a second one with an important dynamical range acts on one of the two ends of the piezoelectric actuator, and a third one acts on the other end of the piezoelectric actuator allowing a fine tuning of the laser frequency on the cavity resonance frequency.

\section{Signal analysis}

In fig. 4 we show the basic principle of the apparatus following ref. [12.

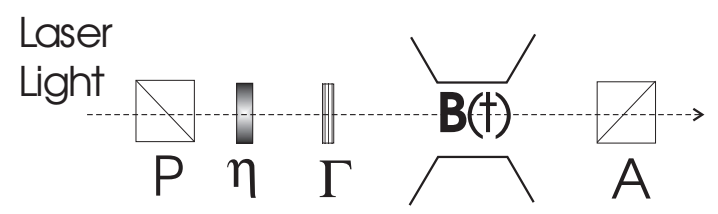

Fig. 4. Basic principle of the detection technique.

A laser beam is polarized by the polarizer P. Let's assume that the light intensity after $\mathrm{P}$ is $I_{t}$. Let's also assume that the light intensity after analyzer $\mathrm{A}$ is $I_{e}$. Going from $\mathrm{P}$ to $\mathrm{A}$, light acquires an ellipticity $\eta(t)$ thanks to an ellipticity modulator, a static ellipticity $\Gamma$, and an ellipticity to be measured $\Psi(t)$. In our case $\Psi(t) \propto B(t)^{2}$. The detection technique suggested in ref. [12] is the heterodyne one. The same technique has been used in the experiences of ref. [17] and [19].

At the extinction $\sigma^{2}$, when $\mathrm{A}$ is crossed with respect to $\mathrm{P}, I_{e}$ can be written as

$$
I_{e}(t)=I_{t} \sigma^{2}+I_{t}[\eta(t)+\Gamma+\Psi(t)]^{2}
$$

For the sake of simplicity, let us now assume that $\eta(t)=\eta_{0} \cos \left(2 \pi \Omega_{m} t+\theta_{m}\right)$ and $\Psi(t)=\Psi_{0} \cos \left(2 \pi \Omega_{e} t+\theta_{e}\right)$, with $\Omega_{m} \gg \Omega_{e}$. It is straightforward to show that $I_{e}$ is constituted by the frequency components given in table 1 :

\begin{tabular}{|c|c|c|c|}
\hline frequency & component & amplitude & phase \\
\hline $\mathrm{DC}$ & $I_{D C}$ & $I_{t}\left(\sigma^{2}+\eta_{0}^{2} / 2+\Psi_{0}^{2} / 2+\Gamma^{2}\right)$ & 0 \\
$\Omega_{e}$ & $I_{\Omega_{e}}$ & $I_{t}\left(2 \Gamma \Psi_{0}\right)$ & $\theta_{e}$ \\
$2 \Omega_{e}$ & $I_{2 \Omega_{e}}$ & $I_{t}\left(\Psi_{0}^{2} / 2\right)$ & $2 \theta_{e}$ \\
$\Omega_{m}$ & $I_{\Omega_{m}}$ & $I_{t}\left(2 \Gamma \eta_{0}\right)$ & $\theta_{m}$ \\
$\Omega_{m} \pm \Omega_{e}$ & $I_{\Omega_{m} \pm \Omega_{e}}$ & $I_{t}\left(\eta_{0} \Psi_{0}\right)$ & $\theta_{m} \pm \theta_{e}$ \\
$2 \Omega_{m}$ & $I_{2 \Omega_{m}}$ & $I_{t}\left(\eta_{0}^{2} / 2\right)$ & $2 \theta_{m}$ \\
\hline
\end{tabular}

Table 1. Frequency components of the signal $I_{e}$ (see eq. 24)

The existence of the two $I_{\Omega_{m} \pm \Omega_{e}}$ components is typical of the heterodyne technique in which one beats the effect to be measured with a carrier effect, $\eta(t)$ in our case. $I_{\Omega_{m}} \pm \Omega_{e}$ components have a linear dependence on the effect for the detected signal. Let us note that the component $I_{\Omega_{e}}$ linear in $\Psi_{0}$ exists even if $\eta(t)=0$ because of the existence of the spurious static ellipticity $\Gamma$. Detecting the signal only by modulating the effect is an example of what is called homodyne technique. Homodyne technique has the advantage of demanding a simpler optical apparatus compared to the heterodyne technique because of the absence of the ellipticity modulator. Homodyne detection is the technique we have chosen for the BMV experiment. As we show in the following paragraph, homodyne detection is particularly interesting when $\Gamma^{2} \gg \sigma^{2}$. This is the case for our BMV experiment. Actually, $\sigma^{2} \approx 10^{-8}$ while $\Gamma^{2} \approx 10^{-4} . \Gamma$ is due to the birefringence of the mirrors that constitute our Fabry-Perot cavity.

\subsection{Comparison between heterodyne technique and homodyne technique}

In the following we will justify our choice by comparing the heterodyne technique and the homodyne one. In particular we calculate the sensitivity expected using the two techniques. We prove that they are completely equivalent with respect to this very important parameter.

In the case of the heterodyne technique one has

$$
\Psi_{0}=\sqrt{\frac{I_{\Omega_{m} \pm \Omega_{e}}^{2}}{2 I_{t} I_{2 \Omega_{m}}}}
$$

The homodyne technique, corresponding to $\eta_{0}=0$, becomes interesting when $\Gamma^{2} \gg \sigma^{2}, \Psi_{0}^{2} / 2$. In this case

$$
\Psi_{0}=\sqrt{\frac{I_{\Omega_{e}}^{2}}{4 I_{t} I_{D C}}}
$$

The limiting noise of both techniques is due to the corpuscular nature of light (shot noise). Shot noise is proportional to the square root of the number of photons detected by the photodiode after the prism A. This is essentially proportional to $I_{D C}$.

$$
i_{\text {shotnoise }}=\sqrt{\frac{2 e^{2} q I_{t}\left(\sigma^{2}+\eta_{0}^{2} / 2+\Psi_{0}^{2} / 2+\Gamma^{2}\right)}{h \nu}}
$$


where $e$ is the absolute value of the electron charge, $q$ is the quantum efficiency of the photodiode, $h$ is the Planck's constant, and $\nu$ is the frequency of light. The rate of photons corresponding to the signal is proportional to $I_{\Omega_{m} \pm \Omega_{e}}$ in the case of heterodyne technique or to $I_{\Omega_{e}}$ in the case of the homodyne technique. The signal-to-noise ratio can be written as

$$
r_{\text {hetero }}=\frac{i_{\text {signal }}}{i_{\text {shotnoise }}}=\sqrt{\frac{2 q I_{t} \Psi_{0}^{2} \eta_{0}^{2}}{\left(\sigma^{2}+\eta_{0}^{2} / 2+\Psi_{0}^{2} / 2+\Gamma^{2}\right) h \nu}}
$$

(where the factor 2 in the square root takes into account the existence of two sidebands) and

$$
r_{\text {homo }}=\frac{i_{\text {signal }}}{i_{\text {shotnoise }}}=\sqrt{\frac{4 q I_{t} \Psi_{0}^{2} \Gamma^{2}}{\left(\sigma^{2}+\Psi_{0}^{2} / 2+\Gamma^{2}\right) h \nu}}
$$

respectively.

It is therefore clear that optimal working conditions thus imply that $\eta_{0}^{2}$ dominates in the $I_{D C}$ component for the heterodyne technique and that $\Gamma^{2}$ dominates in the $I_{D C}$ component for the homodyne technique. In this case, let us finally obtain an expression for the sensitivity $\Psi_{\text {hete }}^{s}$ and $\Psi_{\text {homo }}^{s}$ respectively. For this we impose the condition of a signal-to-noise ratio equal to one. We get

$$
\begin{gathered}
\Psi_{\text {hetero }}^{s}=\sqrt{\frac{h \nu}{2 q I_{t}}} \\
\Psi_{\text {homo }}^{s}=\sqrt{\frac{h \nu}{4 q I_{t}}}
\end{gathered}
$$

The shot noise limit is hardly ever reached. To get a more general expression we note that at optimal working conditions equations 25 and 26 can also be written as

$$
\begin{gathered}
\Psi_{0}=\eta_{0} \frac{I_{\Omega_{m} \pm \Omega_{e}}}{2 I_{D C}} \\
\Psi_{0}=\Gamma \frac{I_{\Omega_{e}}}{I_{D C}}
\end{gathered}
$$

respectively. In the following, we introduce the residual ellipticity noise $\gamma(\Omega)$ that is the noise signal $I_{\text {noise }}(\Omega)$ due to the existence of $\Gamma$ divided by $I_{D C}$. This quantity can be measured by looking at the Fourier spectrum of the signal detected by the photodiode after the analyzer A set at maximum extinction when $\eta_{0}=0$ and no effect is present. We assume that $\eta(t)$ does not add any extra ellipticity noise. Now, by imposing a signal-to-noise ratio equal to one, equations 32 and 33 can be written as

$$
\begin{gathered}
\Psi_{\text {hete }}^{s}=\eta_{0} \frac{I_{\Omega_{m}} \gamma\left(\Omega_{e}\right)}{2 I_{D C}}=\Gamma \frac{2 I_{D C} \gamma\left(\Omega_{e}\right)}{2 I_{D C}}=\Gamma \gamma\left(\Omega_{e}\right) \\
\Psi_{\text {homo }}^{s}=\Gamma \frac{I_{D C} \gamma\left(\Omega_{e}\right)}{I_{D C}}=\Gamma \gamma\left(\Omega_{e}\right)
\end{gathered}
$$

respectively, and we find that the heterodyne and homodyne techniques are completely equivalent. It is also clear that a sensitivity limited by the shot noise can only be reached if $\gamma\left(\Omega_{e}\right)$ is small enough. In the case of the homodyne technique

$$
\gamma\left(\Omega_{e}\right)<\sqrt{\frac{h \nu}{4 q I_{t} \Gamma^{2}}}
$$

To obtain a $\Psi_{\text {homo }}^{s} \simeq 10^{-9} \mathrm{rad} \mathrm{Hz} \mathrm{Hz}^{-1 / 2}$, if $\Gamma \simeq 10^{-2}$ $\operatorname{rad}, \gamma\left(\Omega_{e}\right)<10^{-7} \mathrm{~Hz}^{-1 / 2}$.

The heterodyne technique needs an external modulation of the magnetic field and of the ellipticity which is more demanding. In our experiment we have chosen the homodyne technique $(\eta(t)=0)$ thanks to our pulsed field which gives us a intrinsic modulation.

\subsection{Balanced polarimetry}

A modified version of the apparatus shown in fig. 4 has also been proposed to measure small ellipticities and in particular vacuum magnetic birefringence [30. A quarterwave plate is inserted between the polarizer and the analyzer. The wave plate is set in such a way that the intensity of the ordinary and of the extraordinary ray exiting the analyzer are equal when no birefringence exists along the light path. The presence of an ellipticity in the beam reaching the analyzer prism unbalances the intensities of these two rays. The two ray intensities are measured by two identical photodiodes. The difference of these two intensities gives the final signal for the analysis. This detection method is usually called balanced polarimetry.

One can show that this method is in principle totally equivalent to the one proposed in ref. [12. The electronic extinction given by the substraction of the electric signals corresponding to the intensities of the two rays exiting the analyzer prism play the role of the optical extinction $\sigma^{2}$. The presence of a static uncompensated ellipticity $\Gamma$ gives a $D C$ component to the signal, as any modulated ellipticity gives a corresponding modulation to the signal 30 .

In practice all the results of the previous paragraph apply also for the balanced polarimetry.

\subsection{Correlation between the signal and the magnetic pulse}

As we have shown in the previous paragraphs, the ellipticity can be written as $\Psi(t)=\alpha B(t)^{2}$ or equivalently :

$$
\Psi(t)=\frac{I_{e}(t)-I_{D C}}{2 I_{t} \Gamma},
$$

where $I_{D C}$ has been defined in sec. 3, and we assume that the average effect given in formula 22 can be neglected.

The signal analysis based on the Fourier spectrum discussed in the previous paragraph is the most appropriate only when the ellipticity $\Psi(t)$ is a harmonic function of 
time. As we use a pulsed field, we will use a different technique.

To recover the value of the constant $\alpha$ one can use the following formula

$$
\alpha=\frac{\int_{0}^{T} \Psi(t) B(t)^{2} d t}{\int_{0}^{T} B(t)^{4} d t}
$$

with $B(0)=B(T)=0$.

An interesting property of formula 38 is that if $\Psi(t)$ is proportional to the derivative of $B(t)$ or $B(t)^{2}, \alpha=0$. This means that this technique allows an easy rejection of this kind of spurious signals.

On the other hand, formula 38 gives a value of $\alpha \neq 0$ even if $\Psi(t)$ is not proportional to $B(t)^{2}$. A slightly more complicated formula can be used

$$
\alpha^{\prime}(\tau)=\frac{\int_{0}^{T} \Psi\left(t^{\prime}+\tau\right) B\left(t^{\prime}\right)^{2} d t^{\prime}}{\int_{0}^{T} B\left(t^{\prime}\right)^{4} d t^{\prime}}
$$

It is evident that $\alpha=\alpha^{\prime}(0)$. Now one can compare the function $\alpha^{\prime}(\tau)$ with

$$
\alpha_{B}^{\prime}(\tau)=\frac{\int_{0}^{T} B\left(t^{\prime}+\tau\right)^{2} B\left(t^{\prime}\right)^{2} d t^{\prime}}{\int_{0}^{T} B\left(t^{\prime}\right)^{4} d t^{\prime}}
$$

Only if $\Psi(t)$ is proportional to $B(t)^{2}, \alpha^{\prime}(\tau)$ is proportional to $\alpha_{B}^{\prime}(\tau)$ for any value of $\tau$.

It is important to notice that $I_{e}(t)$ will usually be measured at $\theta=45^{\circ}$ which gives the maximum value for $\Psi(t)$ (see formula 1). Since at $\theta=0^{\circ}, \Psi(t)=0$, even if $B(t) \neq 0$, the measurement of $I_{e}(t)$ at $\theta=0^{\circ}$ and the comparison of it with $I_{D C}$ is a crucial test. Any difference from zero of the quantity $I_{e}\left(t, \theta=0^{\circ}\right)-I_{D C}$ is an indication of noise induced by the magnetic field on the apparatus during the pulse.

\section{Magnet and cryostat}

A first analytical calculation of a model pulsed coil geometry has been presented in ref. 31. A field as high as $25 \mathrm{~T}$ over a meter length seemed to be achievable. This model has been considered as the starting point of the actual coil design in the framework of the BMV project. [32]. The basic idea was to get the current creating the magnetic field as close as possible of the light path over a length as long as possible. For the magnetic birefringence application, one has to maximize the integral of the square of the field over the magnet length $\mathrm{L}$ :

$$
B^{2} L=B_{0}^{2} L_{e q}=\int_{-L / 2}^{L / 2} B_{y}^{2}(z) d z
$$

Here, if $B_{0}$ is the field maximum, we define $L_{e q}$ as the equivalent length of a magnet giving an uniform field value $B_{0}$ on the axis. The ratio $L_{e q} / L$ gives us a measure of the field's uniformity. In fig. 5 we show the configuration we have studied and realized.

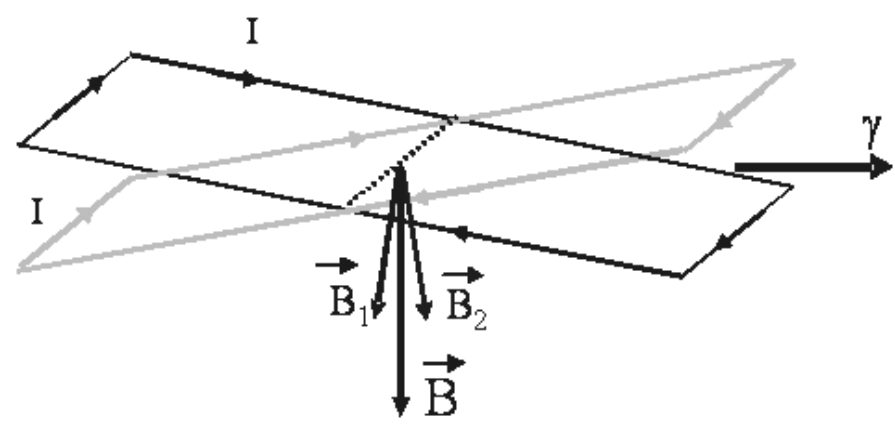

Fig. 5. Scheme of the Xcoil.

Because of the particular geometry, in the following we will call it Xcoil 33 .

In the approximation that the length of the coil is much bigger that its width, one can calculate the field in the centers of the Xcoil by using the expression of the field created by four infinite wires. Following these analytical results, we have realized an actual coil based on the Xcoil scheme. In fig. 6 we can see the comparison between experimental and theoretical field computed by finite element analysis. Here, the field is measured by a pick-up coil and a lock-in amplifier for an oscillating current of $1 \mathrm{~A}$ at $230 \mathrm{~Hz}$.

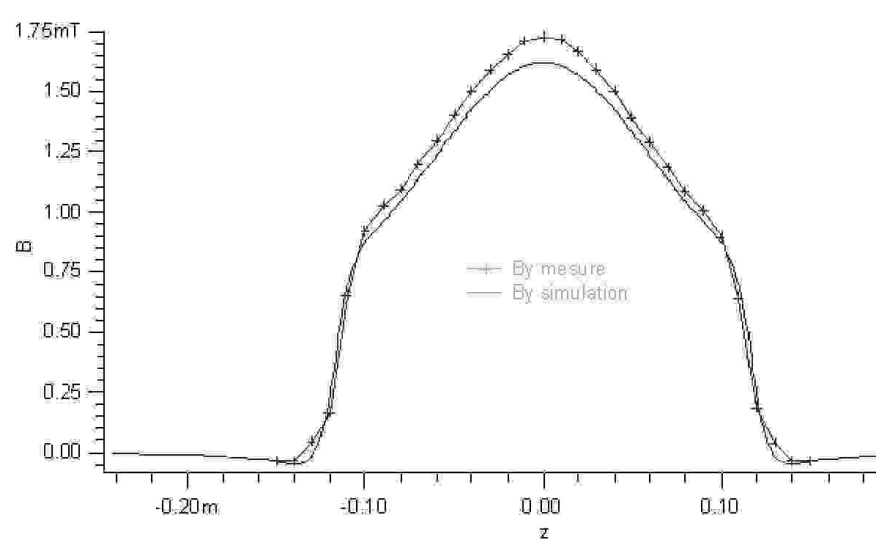

Fig. 6. Computed (line) and measured (line and markers) field profile.

Our parameters leads to a field factor of $1.68 \mathrm{~T} / \mathrm{kA}$, an equivalent length $L_{e q}$ of $13.2 \mathrm{~cm}$ : almost half of the total magnet length. The relevant electrical parameters are $\mathrm{L}=400 \mu H$ and $R_{@ 77 K}=52 \mathrm{~m} \Omega$.

The higher experimental field in fig. 6 is explained by the shape of the wire used that does not exactly correspond to the infinitesimal filaments in the simulation.

The coil support is manufactured using G10, a composite material commonly used to deal with high stresses and cryogenic conditions. External reinforcements from the same material are added after winding to contain the 
magnetic pressure that can be as high as $500 \mathrm{MPa}$ at the field maximum.

We have produced several Xcoils, having outer dimensions of $250 \mathrm{~mm}$ x $100 \times 100 \mathrm{~mm}$. The internal hole available for optical measurements is of $17 \mathrm{~mm}$. This value is mainly given by the dimension of the vacuum tube and thickness of the tubes for double-walled cryostat.

Tests at high field have been performed using a pulsed power supply, that consists of six capacitors switched by thyristors. This capacitor bank is directly down-scaled from the LNCMP's 14 MJ capacitor bank and it containes a maximum energy of $200 \mathrm{~kJ}$.

Due to the increase of resistance by the Joule heating during the shot, the pulse duration is voltage-dependant : it takes $18 \mathrm{~ms}$ at low fields (4T) and $6 \mathrm{~ms}$ at $14 \mathrm{~T}$. Like for conventional pulsed magnets, the coil is placed in a liquid nitrogen cryostat to limit heating consequences. The whole cryostat is double-walled with a vacuum thermal insulation, including for the inner tube. As shown in fig. 7 the cryostat provides two additional functions. It houses the cavity which passes through an opening at room temperature arranged through the cryostat and it allows the cavity to be mechanically disconnected from the coil. Dry and warm nitrogen gas coming from the main bath is reinserted between the cavity and the internal bore of the cryostat in order to prevent any trapping of air moisture. Finally, the cryostat allows for fast disassembly and replacement in the event of degradation of the coil.

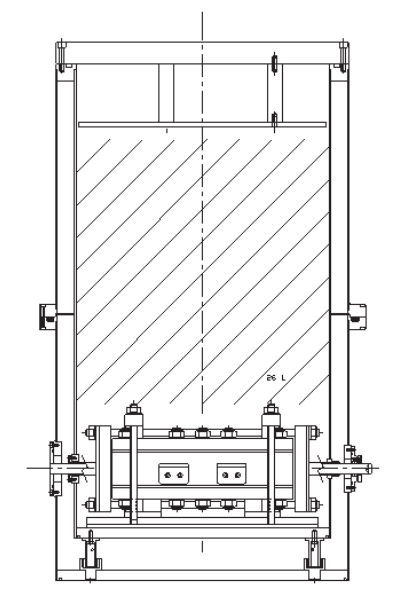

Fig. 7. Drawing of the liquid nitrogen cryostat. The coil is also shown.

In fig. 8 we show a series of magnet pulses obtained by increasing the power supply voltage. The maximum field obtained without generating significant resistance changes in the coil, that would indicate the onset of conductor ageing, has been $14.3 \mathrm{~T}$.

The long-term effect of aging at lower field has been studied by pulsing a coil for 100 times at $11.5 \mathrm{~T}$ and 100 times at $12.5 \mathrm{~T}$. No significant change in its resistance has been detected.

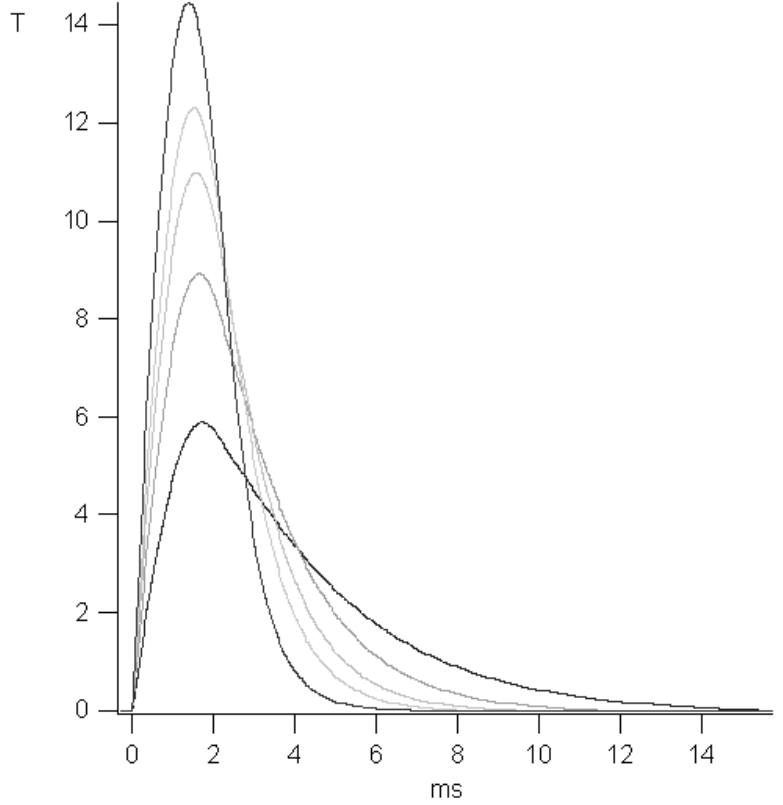

Fig. 8. Series of magnet pulses.

Another crucial point for optical application is that the mechanical noise created by the coil during the pulse has to be as low as possible. We measured for one of our coils, the mechanical noise on the floor of the test area during the pulse. The pulse was $7.5 \mathrm{~T}$ high and its duration was about $5 \mathrm{~ms}$. In fig. 9 we show such a measurement. An interesting feature is that the shock wave arrives after the pulse. The mechanical path was less than one meter.

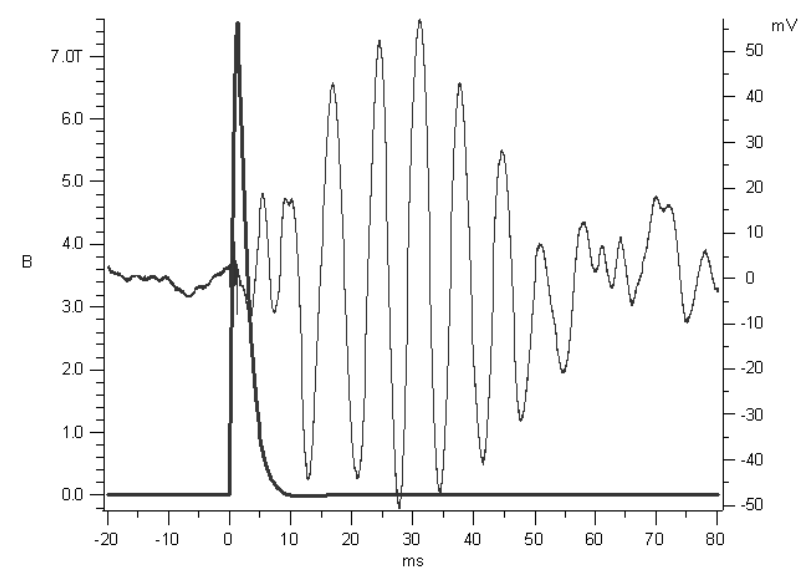

Fig. 9. Mechanical noise during and after a pulse.

During normal operation, by measuring the resistance after each shot, a pick-up signal to monitor the field value and the frequency in vibration spectrum, we can monitor the coil behavior.

Fig. 10] shows a drawing of the experimental set up corresponding to the Fabry-Perot cavity and the field re- 
gion. Two cryostats are shown. The ultra high vacuum chambers for the optics sit on an optical table 3.6 meter long. The length of the cavity is 2.2 meters. The cryostats are supported above the optical table by a structure mechanically decoupled from the optical cavity and the vibrations's path to the mirrors is more than 2 meters long.

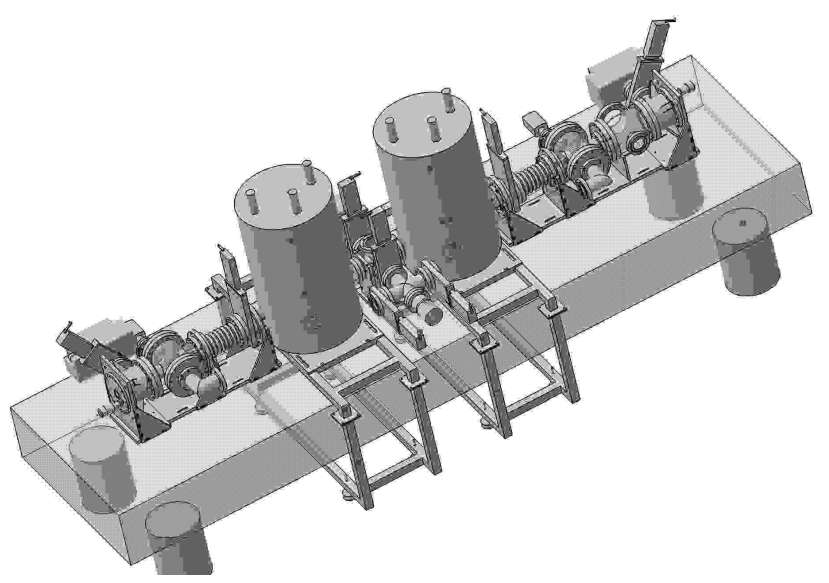

Fig. 10. Drawing of our experimental set up with the two cryostats in place.

\section{Vacuum system}

In fig. 11 we show a scheme of our vacuum system. The most critical parts are the two tubes that allow light to pass through the magnetic field region. Their length is about $50 \mathrm{~cm}$ and the inner diameter is $10 \mathrm{~mm}$.

The main point is that the presence of residual gas in the vacuum pipe induces an ellipticity on the light beam because of the magnetic birefringence of gases (CottonMouton effect) 4. To keep such an ellipticity small compared to the one to be measured, the maximum pressure $P_{g}$ of a residual gas constituent has to satisfy the following formula

$$
P_{g}(a t m) \ll \frac{\lambda \Psi(t)}{2 F L \Delta n_{u} B(t)^{2}}
$$

where following $4 \Delta n_{u}$ is the anisotropy of the index of refraction for the residual gas component for a magnetic field of $1 \mathrm{~T}$ and a pressure of $1 \mathrm{~atm}$ at a temperature of $0{ }^{\circ} \mathrm{C}$. For example, if $\Psi(t) \simeq 10^{-9}, F \simeq 5 \times 10^{5}, L \simeq 1$ $\mathrm{m}, \Delta n_{u} \simeq 10^{-12}$ as for $O_{2}$ gas, $B(t) \simeq 10 \mathrm{~T}, P_{g}(\mathrm{~atm}) \ll$ $7.6 \times 10^{-9}$ torr.

The vacuum system set up allows a dry roughing by a spiral pump and a turbo molecular pump, then a permanent vibration free pumping thanks to two ion pumps. As tests have shown, in the vacuum pipe a pressure better than $10^{-8}$ mbar is expected, while a slightly better pressure should be reached in the vacuum chambers where the mirrors are inserted. Finally, a gas analyzer will be put in between the two vacuum pipe passing through the magnets to check the nature of the residual gas, and monitoring the Cotton-Mouton of the residual gas.

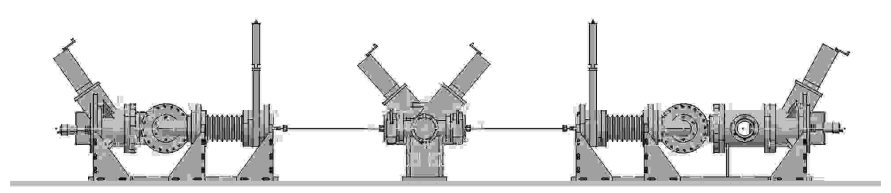

Fig. 11. Scheme of our vacuum system.

\section{Magnetic shielding}

The stray magnetic field of the magnet induces systematic effects on the optics and especially on the mirrors which are the elements closest to the magnets. Mirror Faraday effect, i.e. the rotation of the polarization of a linearly polarized light induced by a magnetic field perpendicular to the mirror surface, has been reported in ref. [40. Faraday rotation was measured being of the order of $K_{F}=3.7 \times 10^{-6}$ $\operatorname{rad} \mathrm{T}^{-1}$ per reflection. Mirror Cotton-Mouton effect, i.e. the ellipticity induced on a linearly polarized light by a magnetic field parallel to the mirror surface, has been reported in ref. 41] and it amounted to about $K_{C M}=10^{-9}$ $\mathrm{T}^{-2}$ per reflection. The mirrors tested in ref. [41] were of different quality than the ones used in ref. 40. Both results depend on the number of reflecting layers and on the layer materials. However they can be used to estimate the maximum stray field tolerable at the mirror location. For example, the ellipticity induced by the mirrors because of their own Cotton-Mouton effect is negligible compared to the ellipticity to be measured, when the stray magnetic field parallel to the mirror surface obeys the following relation.

$$
B_{\text {stray }}^{\|} \ll \sqrt{\frac{\pi}{2 F} \frac{\Psi_{0}}{K_{C M}}}
$$

For example, if $\Psi_{0} \simeq 10^{-9}$ and $F \simeq 500000, B_{\text {stray }}^{\|} \ll$ $2 \times 10^{-3} \mathrm{~T}$.

An equivalent formula can be found for the component of the stray magnetic field perpendicular of the mirror surface.

$$
B_{\text {stray }}^{\perp} \ll \frac{\pi}{2 F} \frac{\rho_{0}}{K_{F}}
$$


where $\rho_{0}$ is the rotation in which one would be interested, and we have assumed as usual that the number of reflections on the mirrors in a Fabry-Perot cavity is equal to $2 F / \pi$.

Using one of our Xcoils, powered for a central field of $7 \mathrm{~T}$, we have measured the stray field on the axis of the coil. At $70 \mathrm{~cm}$ from the coil's center the field is of the order of $1 \mathrm{mT} / \mathrm{kA}$, which corresponds to a reduction of a factor 500 with respect to the field at the centre of the magnet. We have also measured that the shielding factor given by a $4 \mathrm{~mm}$ copper plate is almost 5 at this field. In our experiment, the current is around $8000 \mathrm{~A}$, what gives us a field on the nearest mirror of $0,1 \mathrm{mT}$. With a shielding of at least $80 \%$ (we certainly can expect more than $90 \%$ ), it gives a field on the mirror of $0,02 \mathrm{mT}$. If we need a better shielding, we only need to put a second copper plate. This is a major advantage of pulsed fields over static fields.

\section{Current status and perspectives}

The whole optical system is operational, and the laser has been locked to different cavities. In particular, the laser has been locked to linear cavities (up to $2 \mathrm{~m}$ length) and ring cavities (round trip of $2.4 \mathrm{~m}$ ) of finesses up to 50000 . The measuring technique has been tested by measuring a birefringence induced by an electric field in a gas (Kerr effect) of about $10^{-16}$ [2].

After years of developments and tests, we have finally put optics and magnet together and started debugging and studying sensitivity. First with just one magnet in place, then, when test runs will be completed, with two magnets in place. This configuration corresponds to 40 $\mathrm{T}^{2} \mathrm{~m}$. Finesse greater than 300000 is expected. Sensitivity should also be at least $10^{-8} \mathrm{rad} \mathrm{Hz}^{-1 / 2}$, thanks to the high central frequency of the modulated effect. All these experimental parameters exceed the corresponding values for the PVLAS experiment. Therefore, we will be able to test the PVLAS results using this first version of our apparatus.

Once this first step is accomplished, we will continue towards the QED vacuum magnetic birefringence measurement. The critical points will be to reduce laser noise to reach a sensitivity very close to the quantum limit and to continue the magnet $\mathrm{R} \& \mathrm{D}$ to reach $25 \mathrm{~T}$. A $3-\mathrm{D}$ complete computer modelling of our coils will be implemented to study the behavior of our coils under constraints.

In any case, we will need more powerful transverse pulsed magnets. Xcoils with a field region of $25 \mathrm{~cm}$ have been successfully tested, we are confident that final apparatus will consist of magnets capable to deliver a field over a length such that $B_{0}^{2} L=600 \mathrm{~T}^{2} \mathrm{~m}$. QED ellipticity to be measured will be of the order of $4 \times 10^{-9} \mathrm{rad}$, which should be reached in few hundreds of magnet pulses corresponding to a few weeks of data acquisition.

\section{Acknowledgements}

We thank J. Billette, M. Fouché, D. Forest, J-P. Laurent, J. Mauchain, J-L. Montorio, L. Polizzi, and A. Zitouni. We also acknowledge the strong support of the engineering and technical staff of LCAR, LNCMP and LMA. This work is supported by the ANR-Programme non thématique (ANR-BLAN06-3-139634), and by the $C N R S$ Programme National Astroparticules.

\section{References}

1. J. Kerr, Br. Assoc. Rep. (1901) 568.

2. Q. Majorana, Rendic. Accad. Lincei 11 (1902) 374; Ct. r. hebd. Sanc Acad. Sci. Paris 135 (1902) 159, 235.

3. A. Cotton et H. Mouton, Ct. r. hebd. Séanc Acad. Sci. Paris 141 (1905) 317, 349; Ibid. 142 (1906) 203; Ibid. 145 (1907) 229; Ann. Chem. Phys. 11 (1907) 145, 289.

4. C. Rizzo, A. Rizzo et D. M. Bishop, Int. Rev. Phys. Chem. 16 (1997) 81.

5. W. H. Watson, Proc. Roy. Soc. London A 125 (1929), 345.

6. H. Euler et K. Kochel, Naturwiss. 23 (1935), 246.

7. W. Heisenberg et H. Euler, Z. Phys. 38 (1936), 714.

8. Z. Bialynicka-Birula et I. Bialynicki-Birula, Phys. Rev. D 2 (1970), 2341.

9. S. L. Adler, Ann. Phys. (N. Y.) 87 (1971), 599.

10. C. C. Farr et C. J. Banwell, Proc. Roy. Soc. London A 137 (1932), 275.

11. C. J. Banwell et C. C. Farr, Proc. Roy. Soc. London A 175 (1940), 1.

12. E. Iacopini et E. Zavattini, Phys. Lett. B 85 (1979) 151.

13. L. Maiani, R. Petronzio et E. Zavattini, Phys. Lett. B 175 (1986), 359.

14. R. D. Peccei and H. Quinn, Phys. Rev. Lett. 38, 1440 (1977).

15. G. Raffelt and L. Stodolsky, Phys. Rev. D 37, 1237 (1988). 16. E. Iacopini, B. Smith, G. Stefanini and E. Zavattini, Nuovo Cimento B 61 (1981) 21.

17. R. Cameron, G. Cantatore, A. C. Melissinos, G. Ruoso, Y. Semertzidis, H. Halama, D. Lazarus, A. Prodell, F. Nezrich, C. Rizzo and E. Zavattini, Phys. Rev. D 47 (1993) 3707.

18. D. Bakalov, F. Brandi, G. Cantatore, G. Carugno, S. Carusotto, F. Della Valle, A.M. De Riva, U. Gastaldi, E. Iacopini, P. Micossi, E. Milotti, R. Onofrio, R. Pengo, F. Perrone, G. Petrucci, E. Polacco, C. Rizzo, G. Ruoso, E. Zavattini, G. Zavattini, Quantum Semiclass. Opt. 10 (1998) 239.

19. E. Zavattini, G. Zavattini, G. Ruoso, E. Polacco, E. Milotti, M. Karuza, U. Gastaldi, G. Di Domenico, F. Della Valle, R. Cimino, S. Carusotto, G. Cantatore, and M. Bregant, Phys. Rev. Lett. 96, 110406 (2006).

20. M. Karuza's PhD thesis, University of Padua, Italy (2007), unpublished; see also http://www.sns.ias.edu/ axions/talks/Giovanni_Cantatore.pdf 21. S. Lamoreaux, Nature, 44131 (2006).

22. E. Zavattini et al., arXiv:0706.3419 [hep-ex].

23. K.Zioutas et al. (CAST Collaboration), Phys. Rev. Lett. 94, 121301 (2005).

24. E. Massó, J. Redondo, J. of Cosmology and Astroparticle Physics 9, 15 (2005).

25. http://www.sns.ias.edu/ axions/axions.shtml

26. K. Van Bibber, N.R. Dagderiven, S.E. Koonin, A.K. Kerman, H.N. Nelson, Phys. Rev. Lett. 59, 759 (1987). 
27. http://www.sns.ias.edu/ axions/talks/Pierre_Pugnat.pdf

28. C. Robilliard, R. Battesti, M. Fouché, J. Mauchain, A-M.

Sautivet, F. Amiranoff, and C. Rizzo, Phys. Rev. Lett. in press (arXiv:0707.1296 [hep-ex]).

29. Sheng-Jui Chen, Hsien-Hao Mei, Wei-Tou Ni, arXiv:hep-ex/0611050 [hep-ex].

30. C.Rizzo Europhys. Lett. 41, 483 (1998).

31. S. Askenazy, C. Rizzo, and O. Portugall, Physica B 294295, (2001) 5 .

32. S. Askenazy, J. Billette, P. Dupré, P. Ganau, J. Mackowski, J. Marquez, L. Pinard, O. Portugall, D. Ricard, G.L.J.A. Rikken, C. Rizzo, G. Trénec, J. Vigué, AIP Conf. Proc. 564, (2001) 115.

33. S. Batut et al., submitted to IEEE Trans. Applied Superconductivity.

34. F. Brandi, F. Della Valle, A.M. De Riva, P. Micossi, F. Perrone, C. Rizzo, G. Ruoso, G. Zavattini, Appl. Phys. B 65, 351 (1997).

35. G. Zavattini, G. Cantatore, R. Cimino, G. Di Domenico, F. Della Valle, M. Karuza, E. Milotti, G. Ruoso, Appl. Phys. B 83, 571 (2006).

36. G. Rempe, R.J. Thompson, H.J. Kimble, R. Lalezari, Opt. Lett. 17, 363 (1992).

37. R.V. Pound, Rev. Sci. Instrum. 17, 460 (1946); R.W.P. Drever, J.L. Hall, F.B. Kowalsky, J. Hough, G.M. Ford, A.J. Munley and H. Ward, Appl. Phys. B 31, 97 (1983).

38. G. Cantatore, F.D. Valle, E. Milotti, P. Pace, E. Zavattini, E. Polacco, F. Perrone, C. Rizzo, G. Zavattini, and G. Ruoso, Rev. Sci. Instrum. 66, 2785 (1995).

39. A.M. De Riva, G. Zavattini, S. Marigo, C. Rizzo, G. Ruoso, G. Carugno, R. Onofrio, S. Carusotto, M. Papa, F. Perrone, E. Polacco, G. Cantatore, F. Della Valle, P. Micossi, E. Milotti, P. Pace, E. Zavattini, Rev. Sci. Instrum. 67, 2680 (1996)

40. E. Iacopini, G. Stefanini and E. Zavattini, Appl. Phys. A 32, 63 (1983).

41. G. Bialolenker, E. Polacco, C.Rizzo, G.Ruoso, Appl. Phys. B 68, 703 (1999).

42. F. Bielsa, R. Battesti, C. Robilliard, G. Bialolenker, G. Bailly, G. Trenec, A. Rizzo, C. Rizzo, Eur. Phys. J. D 36, 261 (2005). 